\title{
Well-posedness of delay parabolic equations with unbounded operators acting on delay terms
}

\author{
Allaberen Ashyralyev ${ }^{1,2}$ and Deniz Agirseven ${ }^{3 *}$
}

\section{"Correspondence:}

denizagirseven@trakya.edu.tr

${ }^{3}$ Department of Mathematics,

Trakya University, Edirne, 22030,

Turkey

Full list of author information is

available at the end of the article

\begin{abstract}
In the present paper, the well-posedness of the initial value problem for the delay differential equation $\frac{d v(t)}{d t}+A v(t)=B(t) v(t-\omega)+f(t), t \geq 0 ; v(t)=g(t)(-\omega \leq t \leq 0)$ in an arbitrary Banach space $E$ with the unbounded linear operators $A$ and $B(t)$ in $E$ with dense domains $D(A) \subseteq D(B(t))$ is studied. Two main theorems on well-posedness of this problem in fractional spaces $E_{\alpha}$ are established. In practice, the coercive stability estimates in Hölder norms for the solutions of the mixed problems for delay parabolic equations are obtained.
\end{abstract}

MSC: $35 \mathrm{G} 15$

Keywords: delay parabolic equations; well-posedness; fractional spaces; coercive stability estimates

\section{Introduction}

The stability of delay ordinary differential and difference equations and delay partial differential and difference equations with bounded operators acting on delay terms has been studied extensively in a large cycle of works (see [1-13] and the references therein) and insight has developed over the last three decades. The theory of stability and coercive stability of delay partial differential and difference equations with unbounded operators acting on delay terms has received less attention than delay ordinary differential and difference equations (see [14-19]). It is well known that various initial-boundary value problems for linear evolutionary delay partial differential equations can be reduced to an initial value problem of the form

$$
\left\{\begin{array}{l}
\frac{d v(t)}{d t}+A v(t)=B(t) v(t-\omega)+f(t), \quad t \geq 0 \\
v(t)=g(t) \quad(-\omega \leq t \leq 0)
\end{array}\right.
$$

in an arbitrary Banach space $E$ with the unbounded linear operators $A$ and $B(t)$ in $E$ with dense domains $D(A) \subseteq D(B(t))$. Let $A$ be a strongly positive operator, i.e. $-A$ is the generator of the analytic semigroup $\exp \{-t A\}(t \geq 0)$ of the linear bounded operators with exponentially decreasing norm when $t \rightarrow \infty$. That means the following estimates hold:

$$
\|\exp \{-t A\}\|_{E \mapsto E} \leq M e^{-\delta t}, \quad\|t A \exp \{-t A\}\|_{E \mapsto E} \leq M, \quad t>0
$$

for some $M>1, \delta>0$. Let $B(t)$ be closed operators.

\section{Springer}

O2014 Ashyralyev and Agirseven; licensee Springer. This is an Open Access article distributed under the terms of the Creative Commons Attribution License (http://creativecommons.org/licenses/by/2.0), which permits unrestricted use, distribution, and reproduction in any medium, provided the original work is properly cited. 
A function $v(t)$ is called a solution of the problem (1) if the following conditions are satisfied:

(i) $v(t)$ is continuously differentiable on the interval $[-\omega, \infty)$. The derivative at the endpoint $t=-\omega$ is understood as the appropriate unilateral derivative.

(ii) The element $v(t)$ belongs to $D(A)$ for all $t \in[-\omega, \infty)$, and the function $A v(t)$ is continuous on the interval $[-\omega, \infty)$.

(iii) $v(t)$ satisfies the equation and the initial condition (1).

A solution $v(t)$ of the initial value problem (1) is said to be coercive stable (well-posed)

if

$$
\|A \nu(t)\|_{E} \leq \max _{-\omega \leq t \leq 0}\|A g(t)\|_{E}+\sup _{0 \leq s \leq t}\|f(t)\|_{E}
$$

for every $t,-\omega \leq t<\infty$. We are interested in studying the coercive stability of solutions of the initial value problem under the assumption that

$$
\left\|B(t) A^{-1}\right\|_{E \mapsto E} \leq 1
$$

holds for every $t \geq 0$. We have not been able to obtain the estimate (3) in the arbitrary Banach space $E$. Nevertheless, we can establish the analog of estimates (3) where the space $E$ is replaced by the fractional spaces $E_{\alpha}(0<\alpha<1)$ under an assumption stronger than (4). The coercive stability estimates in Hölder norms for the solutions of the mixed problem of the delay differential equations of the parabolic type are obtained.

The present paper is organized as follows. Section 1 is introduction. In Section 2, two main theorems on well-posedness of the initial value problem (1) are established. In Section 3, the coercive stability estimates in Hölder norms for the solutions of the initialboundary value problem for delay parabolic equations are obtained. Finally, Section 4 is our conclusion.

\section{Theorems on well-posedness}

The strongly positive operator $A$ defines the fractional spaces $E_{\alpha}=E_{\alpha}(E, A)(0<\alpha<1)$ consisting of all $u \in E$ for which the following norms are finite:

$$
\|u\|_{E_{\alpha}}=\sup _{\lambda>0}\left\|\lambda^{1-\alpha} A \exp \{-\lambda A\} u\right\|_{E}
$$

We consider the initial value problem (1) for delay differential equations of parabolic type in the space $C\left(E_{\alpha}\right)$ of all continuous functions $v(t)$ defined on the segment $[0, \infty)$ with values in a Banach space $E_{\alpha}$. First, we consider the problem (1) when $A^{-1}$ and $B(t)$ commute, i.e.

$$
A^{-1} B(t) u=B(t) A^{-1} u, \quad u \in D(A) .
$$

Theorem 2.1 Assume that the condition

$$
\left\|B(t) A^{-1}\right\|_{E \mapsto E} \leq \frac{(1-\alpha)}{M 2^{2-\alpha}}
$$


holds for every $t \geq 0$, where $M$ is the constant from (2). Then for every $t,(n-1) \omega \leq t \leq n \omega$, $n=1, \ldots$, we have the following coercive stability estimate:

$$
\begin{aligned}
& \left\|v^{\prime}(t)\right\|_{E_{\alpha}}+\|A v(t)\|_{E_{\alpha}} \\
& \quad \leq M(\alpha)\left[\max _{-\omega \leq t \leq 0}\|A g(t)\|_{E_{\alpha}}+\sum_{k=1}^{n-1} \max _{-(k-1) \omega \leq s \leq k \omega}\|f(s)\|_{E_{\alpha}}+\max _{(n-1) \omega \leq s \leq t}\|f(s)\|_{E_{\alpha}}\right],
\end{aligned}
$$

where $M(\alpha)$ does not depend on $g(t)$ and $f(t)$. Here, we put $\sum_{k=1}^{m} a_{k}=0$ when $m<1$.

Proof It is clear that

$$
v(t)=u(t)+w(t)
$$

where $u(t)$ is the solution of the problem

$$
\left\{\begin{array}{l}
\frac{d u(t)}{d t}+A u(t)=B(t) u(t-\omega), \quad t \geq 0 \\
u(t)=g(t) \quad(-\omega \leq t \leq 0),
\end{array}\right.
$$

and $w(t)$ is the solution of the problem

$$
\left\{\begin{array}{l}
\frac{d w(t)}{d t}+A w(t)=B(t) w(t-\omega)+f(t), \quad t \geq 0 \\
w(t)=0 \quad(-\omega \leq t \leq 0)
\end{array}\right.
$$

First, we consider the problem (9). Using the formula

$$
u(t)=\exp \{-t A\} g(0)+\int_{0}^{t} \exp \{-(t-s) A\} B(s) g(s-\omega) d s,
$$

the semigroup property, condition (5), and the estimates (2), (6), we obtain

$$
\begin{aligned}
\lambda^{1-\alpha} \| & A \exp \{-\lambda A\} A v(t) \|_{E} \\
\leq & \lambda^{1-\alpha}\|A \exp \{-(\lambda+t) A\} A g(0)\|_{E} \\
& +\lambda^{1-\alpha} \int_{0}^{t}\left\|A \exp \left\{-\frac{\lambda+t-s}{2} A\right\}\right\|_{E \rightarrow E}\left\|B(s) A^{-1}\right\|_{E \rightarrow E} \\
& \times\left\|A \exp \left\{-\frac{\lambda+t-s}{2} A\right\} A g(s-\omega)\right\|_{E} d s \\
\leq & \frac{\lambda^{1-\alpha}}{(\lambda+t)^{1-\alpha}}\|A g(0)\|_{E_{\alpha}}+\frac{1-\alpha}{M 2^{2-\alpha}} \int_{0}^{t} \frac{M \lambda^{1-\alpha} 2^{2-\alpha}}{(\lambda+t-s)^{2-\alpha}} d s \max _{0 \leq s \leq \omega}\|A g(s-\omega)\|_{E_{\alpha}} \\
\leq & \max _{-\omega \leq t \leq 0}\|A g(t)\|_{E_{\alpha}}
\end{aligned}
$$

for every $t, 0 \leq t \leq \omega$ and $\lambda, \lambda>0$. This shows that

$$
\|A u(t)\|_{E_{\alpha}} \leq \max _{-\omega \leq t \leq 0}\|A g(t)\|_{E_{\alpha}}
$$


for every $t, 0 \leq t \leq \omega$. Applying mathematical induction, one can easily show that it is true for every $t$. Namely, assume that the inequality

$$
\|A u(t)\|_{E_{\alpha}} \leq \max _{-\omega \leq s \leq 0}\|A g(s)\|_{E_{\alpha}}
$$

is true for $t,(n-1) \omega \leq t \leq n \omega, n=1,2,3, \ldots$ for some $n$. Letting $t=s+n \omega$, we have

$$
\frac{d u(s+n \omega)}{d s}+A u(s+n \omega)=B(s+n \omega) u(s+(n-1) \omega), \quad 0 \leq s \leq \omega .
$$

Using the estimate (12), we obtain

$$
\max _{n \omega \leq s \leq(n+1) \omega}\|A u(s-\omega)\|_{E_{\alpha}} \leq \max _{-(n-1) \omega \leq t \leq n \omega}\|A u(t)\|_{E_{\alpha}}
$$

for every $t, n \omega \leq t \leq(n+1) \omega, n=1,2,3, \ldots$ and $\lambda, \lambda>0$. This shows that

$$
\|A u(t)\|_{E_{\alpha}} \leq \max _{-\omega \leq t \leq 0}\|A g(t)\|_{E_{\alpha}}
$$

for every $t, n \omega \leq t \leq(n+1) \omega, n=1,2,3, \ldots$. Therefore

$$
\|A u(t)\|_{E_{\alpha}} \leq \max _{-\omega \leq t \leq 0}\|A g(t)\|_{E_{\alpha}}
$$

is true for every $t \geq 0$. Applying (9), the triangle inequality, condition (5), and the estimates (6) and (13), we get

$$
\begin{aligned}
\left\|u^{\prime}(t)\right\|_{E_{\alpha}} & \leq\|A u(t)\|_{E_{\alpha}}+\left\|B(t) A^{-1}\right\|_{E \mapsto E}\|A u(t-\omega)\|_{E_{\alpha}} \\
& \leq\left(1+\frac{1-\alpha}{M 2^{2-\alpha}}\right) \max _{-\omega \leq t \leq 0}\|A g(t)\|_{E_{\alpha}}
\end{aligned}
$$

for every $t \geq 0$. Second, we consider the problem (10). To prove the theorem it suffices to establish the following stability inequality:

$$
\|A w(t)\|_{E_{\alpha}} \leq \frac{M 2^{2-\alpha}}{1-\alpha}\left[\sum_{k=1}^{n-1} \max _{-(k-1) \omega \leq s \leq k \omega}\|f(s)\|_{E_{\alpha}}+\max _{(n-1) \omega \leq s \leq t}\|f(s)\|_{E_{\alpha}}\right]
$$

for the solution of the problem (10) for every $t,(n-1) \omega \leq t \leq n \omega, n=1, \ldots$. Using the formula

$$
w(t)=\int_{0}^{t} \exp \{-(t-s) A\} f(s) d s
$$

the semigroup property, and the definition of the spaces $E_{a}$, we obtain

$$
\begin{aligned}
& \lambda^{1-\alpha}\|A \exp \{-\lambda A\} A w(t)\|_{E} \\
& \quad \leq \lambda^{1-\alpha} \int_{0}^{t}\left\|A^{2} \exp \{-(\lambda+t-s) A\} f(s)\right\|_{E}
\end{aligned}
$$




$$
\begin{aligned}
& \leq M 2^{2-\alpha} \int_{0}^{t} \frac{\lambda^{1-\alpha}}{(\lambda+t-s)^{2-\alpha}}\|f(s)\|_{E_{\alpha}} d s \\
& \leq M 2^{2-\alpha}\left(\int_{0}^{t} \frac{\lambda^{1-\alpha}}{(\lambda+t-s)^{2-\alpha}} d s\right) \max _{0 \leq s \leq t}\|f(s)\|_{E_{\alpha}} \\
& \leq \frac{M 2^{2-\alpha}}{1-\alpha} \max _{0 \leq s \leq t}\|f(s)\|_{E_{\alpha}}
\end{aligned}
$$

for every $t, 0 \leq t \leq \omega$ and $\lambda, \lambda>0$. This shows that

$$
\|A w(t)\|_{E_{\alpha}} \leq \frac{M 2^{2-\alpha}}{1-\alpha} \max _{0 \leq s \leq t}\|f(s)\|_{E_{\alpha}}
$$

for every $t, 0 \leq t \leq \omega$. Applying mathematical induction, one can easily show that it is true for every $t$. Namely, assume that the inequality (15) is true for $t,(n-1) \omega \leq t \leq n \omega$, $n=1, \ldots$, for some $n$. Using the formula

$$
\begin{aligned}
w(t)= & \exp \{-(t-n \omega) A\} w(n \omega)+\int_{n \omega}^{t} \exp \{-(t-s) A\} B(s) w(s-\omega) d s \\
& +\int_{n \omega}^{t} \exp \{-(t-s) A\} f(s) d s,
\end{aligned}
$$

the semigroup property, the definition of the spaces $E_{a}$, the estimate (2), and condition (6), we obtain

$$
\begin{aligned}
\lambda^{1-\alpha} \| & A \exp \{-\lambda A\} A w(t) \|_{E} \\
\leq & \lambda^{1-\alpha}\|A \exp \{-(\lambda+t-n \omega) A\} A w(n \omega)\|_{E} \\
& +\lambda^{1-\alpha} \int_{n \omega}^{t}\left\|A \exp \left\{-\frac{\lambda+t-s}{2} A\right\}\right\|_{E \rightarrow E}\left\|B(s) A^{-1}\right\|_{E \rightarrow E} \\
& \times\left\|A \exp \left\{-\frac{\lambda+t-s}{2} A\right\} A w(s-\omega)\right\|_{E} d s \\
& +\lambda^{1-\alpha} \int_{n \omega}^{t}\left\|A^{2} \exp \{-(\lambda+t-s) A\} f(s)\right\|_{E} d s \\
\leq & \frac{\lambda^{1-\alpha}}{(\lambda+t-n \omega)^{1-\alpha}}\|A w(n \omega)\|_{E_{\alpha}}+\lambda^{1-\alpha}(1-\alpha) \int_{n \omega}^{t} \frac{1}{(\lambda+t-s)^{2-\alpha}}\|A w(s-\omega)\|_{E_{\alpha}} d s \\
& +M 2^{2-\alpha} \int_{n \omega}^{t} \frac{\lambda^{1-\alpha}}{(\lambda+t-s)^{2-\alpha}}\|f(s)\|_{E_{\alpha}} d s \\
\leq & \left(\frac{\lambda^{1-\alpha}}{(\lambda+t-n \omega)^{1-\alpha}}+\lambda^{1-\alpha}(1-\alpha) \int_{n \omega}^{t} \frac{1}{(\lambda+t-s)^{2-\alpha}} d s\right)_{(n-1) \omega \leq t \leq n \omega}\|A w(t)\|_{E_{\alpha}} \\
\leq & \frac{M 2^{2-\alpha}}{1-\alpha} \max _{(n-1) \omega \leq t \leq n \omega}\left[\sum_{k=1}^{n-1} \max _{-(k-1) \omega \leq s \leq k \omega}\|f(s)\|_{E_{\alpha}}+\max _{(n-1) \omega \leq s \leq t}\|f(s)\|_{E_{\alpha}}\right] \\
& +\frac{M 2^{2-\alpha}}{1-\alpha} \sup _{n \omega \leq s \leq t}\|f(s)\|_{E_{\alpha}}=\frac{M 2^{2-\alpha}}{1-\alpha}\left[\sum_{k=1}^{n} \max _{-(k-1) \omega \leq s \leq k \omega}\|f(s)\|_{E_{\alpha}}+\max _{n \omega \leq s \leq t}\|f(s)\|_{E_{\alpha}}\right]
\end{aligned}
$$


for every $t, n \omega \leq t \leq(n+1) \omega, n=1,2,3, \ldots$ and $\lambda, \lambda>0$. This shows that

$$
\|A w(t)\|_{E_{\alpha}} \leq \frac{M 2^{2-\alpha}}{1-\alpha}\left[\sum_{k=1}^{n} \max _{-(k-1) \omega \leq s \leq k \omega}\|f(s)\|_{E_{\alpha}}+\max _{n \omega \leq s \leq t}\|f(s)\|_{E_{\alpha}}\right]
$$

for every $t, n \omega \leq t \leq(n+1) \omega, n=1,2,3, \ldots$. Applying equation (9), the triangle inequality, and condition (5) and estimates (6) and (19), we get

$$
\begin{aligned}
\left\|w^{\prime}(t)\right\|_{E_{\alpha}} & \leq\|A w(t)\|_{E_{\alpha}}+\left\|B(t) A^{-1}\right\|_{E \mapsto E}\|A w(t-\omega)\|_{E_{\alpha}}+\|f(t)\|_{E_{\alpha}} \\
& \leq\left(2+\frac{M 2^{2-\alpha}}{1-\alpha}\right)\left[\sum_{k=1}^{n} \max _{-(k-1) \omega \leq s \leq k \omega}\|f(s)\|_{E_{\alpha}}+\max _{n \omega \leq s \leq t}\|f(s)\|_{E_{\alpha}}\right]
\end{aligned}
$$

for every $t \geq 0$. This result completes the proof of Theorem 2.1.

Now, we consider the problem (1) when

$$
A^{-1} B(t) x \neq B(t) A^{-1} x, \quad x \in D(A)
$$

for some $t \geq 0$. Note that $A$ is a strongly positive operator in a Banach spaces $E$ iff its spectrum $\sigma(A)$ lies in the interior of the sector of angle $\varphi, 0<2 \varphi<\pi$, symmetric with respect to the real axis, and if on the edges of this sector, $S_{1}=[z=\rho \exp (i \varphi): 0 \leq \rho<\infty]$ and $S_{2}=[z=\rho \exp (-i \varphi): 0 \leq \rho<\infty]$ and outside it the resolvent $(z-A)^{-1}$ is the subject to the bound

$$
\left\|(z-A)^{-1}\right\|_{E \rightarrow E} \leq \frac{M_{1}}{1+|z|}
$$

for some $M_{1}>0$. First of all let us give lemmas from the paper [18] that will be needed in the sequel.

Lemma 2.1 For any $z$ on the edges of the sector,

$$
S_{1}=[z=\rho \exp (i \varphi): 0 \leq \rho<\infty]
$$

and

$$
S_{2}=[z=\rho \exp (-i \varphi): 0 \leq \rho<\infty]
$$

and outside it the estimate

$$
\left\|A(z-A)^{-1} x\right\|_{E} \leq \frac{M_{1}^{\alpha} M^{\alpha}\left(1+M_{1}\right)^{1-\alpha} 2^{(2-\alpha) \alpha}}{\alpha(1-\alpha)(1+|z|)^{\alpha}}\|x\|_{E_{\alpha}}
$$

holds for any $x \in E_{\alpha}$. Here and in the future $M$ and $M_{1}$ are the same constants of the estimates (2) and (20). 
Lemma 2.2 Let for all $s \geq 0$ the operator $B(s) A^{-1}-A^{-1} B(s)$ with domain which coincide with $D(A)$ admit a closure $Q=\overline{B(s) A^{-1}-A^{-1} B(s)}$ bounded in $E$. Then for all $\tau>0$ the following estimate holds:

$$
\begin{aligned}
& \left\|A^{-1}[A \exp \{-\tau A\} B(s)-B(s) A \exp \{-\tau A\}] x\right\|_{E} \\
& \quad \leq \frac{e(\alpha+1) M^{\alpha} M_{1}^{1+\alpha}\left(1+2 M_{1}\right)\left(1+M_{1}\right)^{1-\alpha} 2^{(2-\alpha) \alpha}\|Q\|_{E \mapsto E}\|x\|_{E_{\alpha}}}{\tau^{1-\alpha} \pi \alpha^{2}(1-\alpha)} .
\end{aligned}
$$

Here $Q=\overline{A^{-1}(A B(s)-B(s) A) A^{-1}}$.

Suppose that

$$
\begin{aligned}
& {\overline{\left\|A^{-1}(A B(t)-B(t) A) A^{-1}\right\|_{E \mapsto E}}} \\
& \leq \frac{\pi(1-\alpha)^{2} \alpha^{2} \varepsilon}{e M^{1+\alpha} M_{1}^{1+\alpha}\left(1+2 M_{1}\right)\left(1+M_{1}\right)^{1-\alpha} 2^{2+\alpha-\alpha^{2}}(1+\alpha)}
\end{aligned}
$$

holds for every $t \geq 0$. Here and in the future $\varepsilon$ is some constant, $0 \leq \varepsilon \leq 1$.

The application of Lemmas 2.1 and 2.2 enables us to establish the following fact.

Theorem 2.2 Assume that the condition

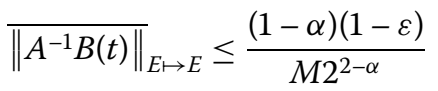

holds for every $t \geq 0$. Then for every $t \geq 0$ the coercive stability estimate (7) holds.

Proof In a similar manner as in the proof of Theorem 2.1 we establish estimates for the solution of the problems (9) and (10), separately. First, we consider the problem (9). Let $0 \leq t \leq \omega$ and $\lambda, \lambda>0$. Then using (11), we have

$$
\begin{aligned}
\lambda^{1-\alpha} & A \exp \{-\lambda A\} A u(t) \\
= & \lambda^{1-\alpha} A \exp \{-(\lambda+t) A\} A g(0) \\
& +\lambda^{1-\alpha} \int_{0}^{t} \exp \left\{-\frac{\lambda+t-s}{2} A\right\} B(s) A \exp \left\{-\frac{\lambda+t-s}{2} A\right\} A g(s-\omega) d s \\
& +\lambda^{1-\alpha} \int_{0}^{t} \exp \left\{-\frac{\lambda+t-s}{2} A\right\}\left[A \exp \left\{-\frac{\lambda+t-s}{2} A\right\} B(s)-B(s) A\right. \\
& \left.\times \exp \left\{-\frac{\lambda+t-s}{2} A\right\}\right] A g(s-\omega) d s \\
= & I_{1}+I_{2}+I_{3},
\end{aligned}
$$

where

$$
\begin{aligned}
& I_{1}=\lambda^{1-\alpha} A \exp \{-(\lambda+t) A\} A g(0), \\
& I_{2}=\lambda^{1-\alpha} \int_{0}^{t} \exp \left\{-\frac{\lambda+t-s}{2} A\right\} B(s) A \exp \left\{-\frac{\lambda+t-s}{2} A\right\} A g(s-\omega) d s,
\end{aligned}
$$




$$
\begin{aligned}
I_{3}= & \lambda^{1-\alpha} \int_{0}^{t} \exp \left\{-\frac{\lambda+t-s}{2} A\right\}\left[A \exp \left\{-\frac{\lambda+t-s}{2} A\right\} B(s)-B(s) A\right. \\
& \left.\times \exp \left\{-\frac{\lambda+t-s}{2} A\right\}\right] A g(s-\omega) d s .
\end{aligned}
$$

Using the estimates (2), (20), and condition (22), we obtain

$$
\begin{aligned}
\left\|I_{1}\right\|_{E}= & \lambda^{1-\alpha}\|A \exp \{-(\lambda+t) A\} A g(0)\|_{E} \\
\leq & \frac{\lambda^{1-\alpha}}{(\lambda+t)^{1-\alpha}}\|A g(0)\|_{E_{\alpha}} \leq \frac{\lambda^{1-\alpha}}{(\lambda+t)^{1-\alpha}} \max _{-\omega \leq t \leq 0}\|A g(t)\|_{E_{\alpha}}, \\
\left\|I_{2}\right\|_{E} \leq & \lambda^{1-\alpha} \int_{0}^{t}\left\|A \exp \left\{-\frac{\lambda+t-s}{2} A\right\}\right\|_{E \mapsto E}\left\|\overline{A^{-1} B(s)}\right\|_{E \mapsto E} \\
& \times\left\|A \exp \left\{-\frac{\lambda+t-s}{2} A\right\} A g(s-\omega)\right\| \|_{E} d s \\
\leq & \max _{0 \leq t \leq \omega}\left\|\overline{A^{-1} B(t)}\right\|_{E \mapsto E} \int_{0}^{t} \frac{M \lambda^{1-\alpha} 2^{2-\alpha}}{(\lambda+t-s)^{2-\alpha}} d s \max _{0 \leq s \leq \omega}\|A g(s-\omega)\|_{E_{\alpha}} \\
\leq & \max _{-\omega \leq t \leq 0}\|A g(t)\|_{E_{\alpha}}\left(1-\frac{\lambda^{1-\alpha}}{(\lambda+t)^{1-\alpha}}\right)(1-\varepsilon)
\end{aligned}
$$

for every $t, 0 \leq t \leq \omega$ and $\lambda, \lambda>0$. Now let us estimate $I_{3}$. By Lemma 2.1 and using the estimate (21), we obtain

$$
\begin{aligned}
& \left\|I_{3}\right\|_{E} \leq \lambda^{1-\alpha} \int_{0}^{t}\left\|A \exp \left\{-\frac{\lambda+t-s}{2} A\right\}\right\|_{E \mapsto E} \\
& \times\left\|A^{-1}\left[A \exp \left\{-\frac{\lambda+t-s}{2} A\right\} B(s)-B(s) A \exp \left\{-\frac{\lambda+t-s}{2} A\right\}\right] A g(s-\omega)\right\|_{E} d s \\
& \leq \lambda^{1-\alpha} e(1+\alpha) M^{1+\alpha} M_{1}^{1+\alpha}\left(1+2 M_{1}\right)\left(1+M_{1}\right)^{1-\alpha} 2^{(2-\alpha) \alpha} \\
& \times \int_{0}^{t} \frac{\left\|\overline{A^{-1}(A B(s)-B(s) A) A^{-1}}\right\|_{E \mapsto E} 2^{2-\alpha}\|A g(s-\omega)\|_{E_{\alpha}}}{(\lambda+t-s)^{2-\alpha} \pi \alpha^{2}(1-\alpha)} d s \\
& \leq \max _{0 \leq s \leq \omega}{\overline{\left\|A^{-1}(A B(s)-B(s) A) A^{-1}\right\|_{E \mapsto E}}} \\
& \times \int_{0}^{t} \frac{\lambda^{1-\alpha} e(1+\alpha) M^{1+\alpha} M_{1}^{1+\alpha}\left(1+2 M_{1}\right)\left(1+M_{1}\right)^{1-\alpha} 2^{(2-\alpha) \alpha} 2^{2-\alpha}}{(\lambda+t-s)^{2-\alpha} \pi \alpha^{2}(1-\alpha)} d s \\
& \times \max _{-\omega \leq t \leq 0}\|A g(t)\|_{E_{\alpha}} \leq \max _{-\omega \leq t \leq 0}\|A g(t)\|_{E_{\alpha}}\left(1-\frac{\lambda^{1-\alpha}}{(\lambda+t)^{1-\alpha}}\right) \varepsilon
\end{aligned}
$$

for every $t, 0 \leq t \leq \omega$ and $\lambda, \lambda>0$. Using the triangle inequality, we obtain

$$
\lambda^{1-\alpha}\|A \exp \{-\lambda A\} A u(t)\|_{E} \leq \max _{-\omega \leq t \leq 0}\|A g(t)\|_{E_{\alpha}}
$$

for every $t, 0 \leq t \leq \omega$ and $\lambda, \lambda>0$. This shows that

$$
\|A u(t)\|_{E_{\alpha}} \leq \max _{-\omega \leq t \leq 0}\|A g(t)\|_{E_{\alpha}}
$$

for every $t, 0 \leq t \leq \omega$. In a similar manner as with Theorem 2.1 applying mathematical induction, one can easily show that it is true for every $t$. Therefore, to prove the theorem 
it suffices to establish the coercive stability inequality (15) for the solution of the problem (10). Now, we consider the problem (10). Exactly in the same manner, using (16), the semigroup property, and the definition of the spaces $E_{a}$, we obtain (15) for every $t, 0 \leq t \leq \omega$. Applying mathematical induction, one can easily show that it is true for every $t$. Namely, assume that the inequality (15) is true for $t,(n-1) \omega \leq t \leq n \omega, n=1, \ldots$ for some $n$. Using (18) and the semigroup property, we write

$$
\begin{aligned}
\lambda^{1-\alpha} A & \exp \{-\lambda A\} A w(t) \\
= & \lambda^{1-\alpha} A \exp \{-(\lambda+t-n \omega) A\} A w(n \omega) \\
& +\lambda^{1-\alpha} \int_{n \omega}^{t} \exp \left\{-\frac{\lambda+t-s}{2} A\right\} B(s) A \exp \left\{-\frac{\lambda+t-s}{2} A\right\} A w(s-\omega) d s \\
& +\lambda^{1-\alpha} \int_{n \omega}^{t} \exp \left\{-\frac{\lambda+t-s}{2} A\right\}\left[A \exp \left\{-\frac{\lambda+t-s}{2} A\right\} B(s)-B(s) A\right. \\
& \left.\times \exp \left\{-\frac{\lambda+t-s}{2} A\right\}\right] A w(s-\omega) d s+\lambda^{1-\alpha} \int_{n \omega}^{t} A^{2} \exp \{-(\lambda+t-s) A\} f(s) d s \\
= & I_{1}+I_{2}+I_{3}+I_{4},
\end{aligned}
$$

where

$$
\begin{aligned}
I_{1}= & \lambda^{1-\alpha} A \exp \{-(\lambda+t-n \omega) A\} A w(n \omega), \\
I_{2}= & \lambda^{1-\alpha} \int_{n \omega}^{t} \exp \left\{-\frac{\lambda+t-s}{2} A\right\} B(s) A \exp \left\{-\frac{\lambda+t-s}{2} A\right\} A w(s-\omega) d s, \\
I_{3}= & \lambda^{1-\alpha} \int_{n \omega}^{t} \exp \left\{-\frac{\lambda+t-s}{2} A\right\}\left[A \exp \left\{-\frac{\lambda+t-s}{2} A\right\} B(s)-B(s) A\right. \\
& \left.\times \exp \left\{-\frac{\lambda+t-s}{2} A\right\}\right] A w(s-\omega) d s, \\
I_{4}= & \lambda^{1-\alpha} \int_{n \omega}^{t} A^{2} \exp \{-(\lambda+t-s) A\} f(s) d s .
\end{aligned}
$$

Using the estimate (2) and condition (22), we obtain

$$
\begin{aligned}
\left\|I_{1}\right\|_{E}= & \lambda^{1-\alpha}\|A \exp \{-(\lambda+t-n \omega) A\} A w(n \omega)\|_{E} \leq \frac{\lambda^{1-\alpha}}{(\lambda+t-n \omega)^{1-\alpha}}\|A w(n \omega)\|_{E_{\alpha}}, \\
\left\|I_{2}\right\|_{E} \leq & \lambda^{1-\alpha} \int_{n \omega}^{t}\left\|A \exp \left\{-\frac{\lambda+t-s}{2} A\right\}\right\|_{E \mapsto E}\left\|\overline{A^{-1} B(s)}\right\|_{E \mapsto E} \\
& \times\left\|A \exp \left\{-\frac{\lambda+t-s}{2} A\right\} A w(s-\omega)\right\|_{E} d s \\
\leq & \max _{n \omega \leq t \leq(n+1) \omega}\left\|\frac{A^{-1} B(t)}{}\right\|_{E \mapsto E} \int_{n \omega}^{t} \frac{M \lambda^{1-\alpha} 2^{2-\alpha}}{(\lambda+t-s)^{2-\alpha}} d s \max _{n \omega \leq s \leq(n+1) \omega}\|A w(s-\omega)\|_{E_{\alpha}} \\
\leq & \left(1-\frac{\lambda^{1-\alpha}}{(\lambda+t-n \omega)^{1-\alpha}}\right)(1-\varepsilon) \max _{(n-1) \omega \leq s \leq n \omega}\|A w(s)\|_{E_{\alpha}}, \\
\left\|I_{4}\right\|_{E} \leq & M 2^{2-\alpha} \int_{n \omega}^{t} \frac{\lambda^{1-\alpha}}{(\lambda+t-s)^{2-\alpha}}\|f(s)\|_{E_{\alpha}} d s \leq \frac{M 2^{2-\alpha}}{1-\alpha} \sup _{n \omega \leq s \leq t}\|f(s)\|_{E_{\alpha}}
\end{aligned}
$$


for every $t, n \omega \leq t \leq(n+1) \omega, n=1,2,3, \ldots$, and $\lambda, \lambda>0$. Now let us estimate $I_{3}$. By Lemma 2.2 and using the estimate (2) and condition (21), we obtain

$$
\begin{aligned}
& \left\|I_{3}\right\|_{E} \leq \lambda^{1-\alpha} \int_{n \omega}^{t}\left\|A \exp \left\{-\frac{\lambda+t-s}{2} A\right\}\right\|_{E \mapsto E} \\
& \times\left\|A^{-1}\left[A \exp \left\{-\frac{\lambda+t-s}{2} A\right\} B(s)-B(s) A \exp \left\{-\frac{\lambda+t-s}{2} A\right\}\right] A w(s-\omega)\right\|_{E} d s \\
& \leq \lambda^{1-\alpha} e(1+\alpha) M^{1+\alpha} M_{1}^{1+\alpha}\left(1+2 M_{1}\right)\left(1+M_{1}\right)^{1-\alpha} 2^{(2-\alpha) \alpha} \\
& \times \int_{n \omega}^{t} \frac{\left\|\overline{A^{-1}(A B(s)-B(s) A) A^{-1}}\right\|_{E \mapsto E} 2^{2-\alpha}\|A w(s-\omega)\|_{E_{\alpha}}}{(\lambda+t-s)^{2-\alpha} \pi \alpha^{2}(1-\alpha)} d s \\
& \leq \max _{0 \leq s \leq \omega} \overline{\left\|A^{-1}(A B(s)-B(s) A) A^{-1}\right\|} \mid E \mapsto E \\
& \times \int_{n \omega}^{t} \frac{\lambda^{1-\alpha} e(1+\alpha) M^{1+\alpha} M_{1}^{1+\alpha}\left(1+2 M_{1}\right)\left(1+M_{1}\right)^{1-\alpha} 2^{(2-\alpha) \alpha} 2^{2-\alpha}}{(\lambda+t-s)^{2-\alpha} \pi \alpha^{2}(1-\alpha)} d s \\
& \times \max _{(n-1) \omega \leq s \leq n \omega}\|A w(s)\|_{E_{\alpha}} \\
& \leq\left(1-\frac{\lambda^{1-\alpha}}{(\lambda+t-n \omega)^{1-\alpha}}\right) \varepsilon \max _{(n-1) \omega \leq s \leq n \omega}\|A w(s)\|_{E_{\alpha}}
\end{aligned}
$$

for every $t, n \omega \leq t \leq(n+1) \omega, n=1,2,3, \ldots$ and $\lambda, \lambda>0$. Using the triangle inequality and estimates for all $\left\|I_{k}\right\|_{E}, k=1,2,3,4$, we obtain

$$
\lambda^{1-\alpha}\|A \exp \{-\lambda A\} A w(t)\|_{E} \leq \frac{M 2^{2-\alpha}}{1-\alpha}\left[\sum_{k=1}^{n} \max _{-(k-1) \omega \leq s \leq k \omega}\|f(s)\|_{E_{\alpha}}+\max _{n \omega \leq s \leq t}\|f(s)\|_{E_{\alpha}}\right]
$$

for every $t, n \omega \leq t \leq(n+1) \omega, n=1,2,3, \ldots$ and $\lambda, \lambda>0$. This shows that

$$
\|A w(t)\|_{E_{\alpha}} \leq \frac{M 2^{2-\alpha}}{1-\alpha}\left[\sum_{k=1}^{n} \max _{-(k-1) \omega \leq s \leq k \omega}\|f(s)\|_{E_{\alpha}}+\max _{n \omega \leq s \leq t}\|f(s)\|_{E_{\alpha}}\right]
$$

for every $t, n \omega \leq t \leq(n+1) \omega, n=1,2,3, \ldots$. This result completes the proof of Theorem 2.2.

Note that these abstract results are applicable to the study of stability of various delay parabolic equations with local and nonlocal boundary conditions with respect to the space variables. However, it is important to study the structure of $E_{\alpha}$ for space operators in Banach spaces. The structure of $E_{\alpha}$ for some space differential and difference operators in Banach spaces has been investigated (see [20-30]). In Section 3, applications of Theorem 2.1 to the study of the coercive stability of initial-boundary value problem for delay parabolic equations are given. 


\section{Applications}

First, we consider the initial-boundary value problem for one dimensional delay differential equations of parabolic type

$$
\left\{\begin{array}{l}
\frac{\partial u(t, x)}{\partial t}-a(x) \frac{\partial^{2} u(t, x)}{\partial x^{2}}+\delta u(t, x) \\
\quad=b(t)\left(-a(x) \frac{\partial^{2} u(t-\omega, x)}{\partial x^{2}}+\delta u(t-\omega, x)\right)+f(t, x), \quad 0<t<\infty, x \in(0, l), \\
u(t, x)=g(t, x), \quad-\omega \leq t \leq 0, x \in[0, l] \\
u(t, 0)=u(t, l)=0, \quad-\omega \leq t<\infty
\end{array}\right.
$$

where $a(x), b(t), g(t, x), f(t, x)$ are given sufficiently smooth functions and $\delta>0$ is a sufficiently large number. We will assume that $a(x) \geq a>0$. The problem (23) has a unique smooth solution. This allows us to reduce the initial-boundary value problem (23) to the initial value problem (1) in Banach space $E=C[0, l]$ with a differential operator $A^{x}$ defined by the formula

$$
A^{x} u=-a(x) \frac{d^{2} u}{d x^{2}}+\delta u
$$

with domain $D\left(A^{x}\right)=\left\{u \in C^{(2)}[0,1]: u(0)=u(1)=0\right\}$. Let us give a number of corollaries of the abstract Theorem 2.1.

\section{Theorem 3.1 Assume that}

$$
\sup _{0 \leq t<\infty}|b(t)| \leq \frac{1-\alpha}{M 2^{2-\alpha}} .
$$

Then for all $t \geq 0$ the solutions of the initial-boundary value problem (23) satisfy the following coercive stability estimates:

$$
\begin{aligned}
& \left\|u^{\prime}(t, \cdot)\right\|_{C^{2 \alpha}[0, l]}+\|u(t, \cdot)\|_{C^{2+2 \alpha}[0, l]} \\
& \leq M(\alpha)\left[\max _{-\omega \leq t \leq 0}\|g(t, \cdot)\|_{C^{2+2 \alpha}[0, l]}\right. \\
& \left.\quad+\sum_{k=1}^{n} \max _{-(k-1) \omega \leq s \leq k \omega}\|f(s, \cdot)\|_{C^{2 \alpha}[0, l]}+\max _{n \omega \leq s \leq t}\|f(s, \cdot)\|_{C^{2 \alpha}[0, l]}\right], \quad 0<\alpha<\frac{1}{2},
\end{aligned}
$$

where $M(\alpha)$ is not dependent on $g(t, x)$ and $f(t, x)$. Here $C^{\beta}[0, l]$ is the space of functions satisfying a Hölder condition with the indicator $\beta \in(0,1)$.

The proof of Theorem 3.1 is based on the estimate

$$
\left\|\exp \left\{-t A^{x}\right\}\right\|_{C[0, l] \rightarrow C[0, l]} \leq M, \quad t \geq 0,
$$

and on the abstract Theorem 2.1, on the strong positivity of the operator $A^{x}$ in $C[0, l]$ (see $[31,32])$ and on Theorem 3.2 on the structure of the fractional space $E_{\alpha}=E_{\alpha}\left(C[0, l], A^{x}\right)$ for $0<\alpha<\frac{1}{2}$.

Theorem 3.2 For $\alpha \in\left(0, \frac{1}{2}\right)$, the norms of the space $E_{\alpha}\left(C[0, l], A^{x}\right)$ and the Hölder space $C^{2 \alpha}[0, l]$ are equivalent $[21]$. 
Second, we consider the initial nonlocal boundary value problem for one dimensional delay differential equations of parabolic type,

$$
\left\{\begin{array}{l}
\frac{\partial u(t, x)}{\partial t}-a(x) \frac{\partial^{2} u(t, x)}{\partial x^{2}}+\delta u(t, x) \\
\quad=b(t)\left(-a(x) \frac{\partial^{2} u(t-\omega, x)}{\partial x^{2}}+\delta u(t-\omega, x)\right)+f(t, x), \quad 0<t<\infty, x \in(0, l), \\
u(t, x)=g(t, x), \quad-\omega \leq t \leq 0, x \in[0, l], \\
u(t, 0)=u(t, l), \quad u_{x}(t, 0)=u_{x}(t, l), \quad-\omega \leq t<\infty
\end{array}\right.
$$

where $a(x), b(t), g(t, x), f(t, x)$ are given sufficiently smooth functions and $\delta>0$ is a sufficiently large number. We will assume that $a(x) \geq a>0$. The problem (26) has a unique smooth solution. This allows us to reduce the initial-boundary value problem (26) to the initial value problem (1) in Banach space $E=C[0, l]$ with a differential operator $A^{x}$ defined by the formula

$$
A^{x} u=-a(x) \frac{d^{2} u}{d x^{2}}+\delta u
$$

with domain $D\left(A^{x}\right)=\left\{u \in C^{(2)}[0,1]: u(0)=u(1), u^{\prime}(0)=u^{\prime}(1)\right\}$. Let us give a number of corollaries of the abstract Theorem 2.1.

Theorem 3.3 Assume that condition (25) holds. Then for all $t \geq 0$ the solutions of the initial-boundary value problem (26) satisfy the following coercive stability estimates:

$$
\begin{aligned}
& \left\|u^{\prime}(t, \cdot)\right\|_{C^{2 \alpha}[0, l]}+\|u(t, \cdot)\|_{C^{2+2 \alpha}[0, l]} \\
& \quad \leq M(\alpha)\left[\max _{-\omega \leq t \leq 0}\|g(t, \cdot)\|_{C^{2+2 \alpha}[0, l]}\right. \\
& \left.\quad+\sum_{k=1}^{n} \max _{-(k-1) \omega \leq s \leq k \omega}\|f(s, \cdot)\|_{C^{2 \alpha}[0, l]}+\max _{n \omega \leq s \leq t}\|f(s, \cdot)\|_{C^{2 \alpha}[0, l]}\right], \quad 0<\alpha<\frac{1}{2},
\end{aligned}
$$

where $M(\alpha)$ is not dependent on $g(t, x)$ and $f(t, x)$.

The proof of Theorem 3.3 is based on the estimate

$$
\left\|\exp \left\{-t A^{x}\right\}\right\|_{C[0, l] \rightarrow C[0, l]} \leq M, \quad t \geq 0
$$

and on the abstract Theorem 2.1, on the strong positivity of the operator $A^{x}$ in $C[0, l]$ (see [6]) and on Theorem 3.4 on the structure of the fractional space $E_{\alpha}=E_{\alpha}\left(C[0, l], A^{x}\right)$ for $0<\alpha<\frac{1}{2}$.

Theorem 3.4 For $\alpha \in\left(0, \frac{1}{2}\right)$, the norms of the space $E_{\alpha}\left(C[0, l], A^{x}\right)$ and the Hölder space $C^{2 \alpha}[0, l]$ are equivalent $[6]$.

Third, we consider the initial value problem on the range

$$
\left\{0 \leq t \leq 1, x=\left(x_{1}, \ldots, x_{n}\right) \in \mathbb{R}^{n}, r=\left(r_{1}, \ldots, r_{n}\right)\right\}
$$


for $2 m$ th order multidimensional delay differential equations of parabolic type,

$$
\left\{\begin{array}{l}
\frac{\partial u(t, x)}{\partial t}+\sum_{|r|=2 m} a_{\tau}(x) \frac{\partial|r|}{\partial x_{1}^{r_{1}} \ldots(t, x)} x_{n}^{r_{n}}+\delta u(t, x) \\
\quad=b(t)\left(\sum_{|r|=2 m} a_{\tau}(x) \frac{\partial|r|}{\partial x_{1}^{r_{1}} \ldots \partial x_{n}}+\delta\right) \\
u(t, x)=g(t, x), \quad-\omega \leq t \leq 0, x \in \mathbb{R}^{n},|r|=r_{1}+\cdots+r_{n},
\end{array}\right.
$$

where $a_{r}(x), b(t), g(t, x)$, and $f(t, x)$ are sufficiently smooth functions and $\delta>0$ is a sufficiently large number. We will assume that the symbol $\left[\xi=\left(\xi_{1}, \ldots, \xi_{n}\right) \in \mathbb{R}^{n}\right]$

$$
A_{1}^{x}(\xi)=\sum_{|r|=2 m} a_{r}(x)\left(i \xi_{1}\right)^{r_{1}} \cdots\left(i \xi_{n}\right)^{r_{n}}
$$

of the differential operator of the form

$$
A_{1}^{x}=\sum_{|r|=2 m} a_{r}(x) \frac{\partial^{|r|}}{\partial x_{1}^{r_{1}} \cdots \partial x_{n}^{r_{n}}}
$$

acting on functions defined on the space $\mathbb{R}^{n}$, satisfies the inequalities

$$
0<M_{1}|\xi|^{2 m} \leq(-1)^{m} A_{1}^{x}(\xi) \leq M_{2}|\xi|^{2 m}<\infty
$$

for $\xi \neq 0$, where $|\xi|=\sqrt{\left|\xi_{1}\right|^{2}+\cdots+\left|\xi_{n}\right|^{2}}$. The problem (28) has a unique smooth solution. This allows us to reduce the initial value problem (28) to the initial value problem (1) in Banach space $E$ with a strongly positive operator $A^{x}=A_{1}^{x}+\delta I$ defined by (29). Let us give a number of corollaries of the abstract Theorem 2.1.

Theorem 3.5 Assume that condition (25) holds. Then for all $t \geq 0$ the solutions of the initial-boundary value problem (28) satisfy the following coercive stability estimates:

$$
\begin{aligned}
& \left\|u^{\prime}(t, \cdot)\right\|_{C^{2 m \alpha}\left(\mathbb{R}^{n}\right)}+\sum_{|r|=2 m}\left\|\frac{\partial^{|r|} u(t, \cdot)}{\partial x_{1}^{r_{1}} \cdots \partial x_{n}^{r_{n}}}\right\|_{C^{2 m \alpha}\left(\mathbb{R}^{n}\right)} \\
& \leq M(\alpha)\left[\max _{-\omega \leq t \leq 0} \sum_{|r|=2 m}\left\|\frac{\partial^{|r|} g(t, \cdot)}{\partial x_{1}^{r_{1}} \cdots \partial x_{n}^{r_{n}}}\right\|_{C^{2 m \alpha\left(\mathbb{R}^{n}\right)}}\right. \\
& \left.\quad+\sum_{k=1}^{n} \max _{-(k-1) \omega \leq s \leq k \omega}\|f(s, \cdot)\|_{C^{2 m \alpha}\left(\mathbb{R}^{n}\right)}+\max _{n \omega \leq s \leq t}\|f(s, \cdot)\|_{C^{2 m \alpha}\left(\mathbb{R}^{n}\right)}\right], \quad 0<\alpha<\frac{1}{2 m},
\end{aligned}
$$

where $M_{2}(\alpha)$ does not depend on $g(t, x)$ and $f(t, x)$. Here $C^{\varepsilon}\left(\mathbb{R}^{n}\right)$ is the space of functions satisfying a Hölder condition with the indicator $\varepsilon \in(0,1)$.

The proof of Theorem 3.5 is based on the estimate

$$
\left\|\exp \left\{-t A^{x}\right\}\right\|_{C\left(\mathbb{R}^{n}\right) \rightarrow C\left(\mathbb{R}^{n}\right)} \leq M, \quad t \geq 0,
$$

and on the abstract Theorem 2.1, on the strong positivity of the operator $A^{x}$ in $C\left(\mathbb{R}^{n}\right)$, and on the equivalence of the norms in the spaces $E_{\alpha}=E_{\alpha}\left(A, C\left(\mathbb{R}^{n}\right)\right)$ and $C^{2 m \alpha}\left(\mathbb{R}^{n}\right)$ when $0<\alpha<\frac{1}{2 m}[20,23]$. 


\section{Conclusion}

In the present paper, two theorems on the well-posedness of the initial value problem for the delay parabolic differential equations with unbounded operators acting on delay terms in fractional spaces $E_{\alpha}$ are established. In practice, the coercive stability estimates in Hölder norms for the solutions of the mixed problems for delay parabolic equations are obtained.

\section{Competing interests}

The authors declare that they have no competing interests.

\section{Authors' contributions}

All authors read and approved the final manuscript.

\section{Author details}

${ }^{1}$ Department of Mathematics, Fatih University, Istanbul, 34500, Turkey. ${ }^{2}$ Department of Mathematics, ITTU, Gerogly Street, Ashgabat, 74400, Turkmenistan. ${ }^{3}$ Department of Mathematics, Trakya University, Edirne, 22030, Turkey.

\section{Acknowledgements}

This work is supported by Trakya University Scientific Research Projects Unit (Project No: 2010-91).

Received: 31 March 2014 Accepted: 8 May 2014 Published: 20 May 2014

\section{References}

1. Al-Mutib, AN: Stability properties of numerical methods for solving delay differential equations. J. Comput. Appl. Math. 10(1), 71-79 (1984)

2. Bellen, A: One-step collocation for delay differential equations. J. Comput. Appl. Math. 10(3), 275-283 (1984)

3. Bellen, A, Jackiewicz, Z, Zennaro, M: Stability analysis of one-step methods for neutral delay-differential equations. Numer. Math. 52(6), 605-619 (1988)

4. Cooke, KL, Györi, I: Numerical approximation of the solutions of delay differential equations on an infinite interval using piecewise constant arguments. Comput. Math. Appl. 28, 81-92 (1994)

5. Torelli, L: Stability of numerical methods for delay differential equations. J. Comput. Appl. Math. 25, 15-26 (1989)

6. Yenicerioglu, AF, Yalcinbas, S: On the stability of the second-order delay differential equations with variable coefficients. Appl. Math. Comput. 152(3), 667-673 (2004)

7. Yenicerioglu, AF: Stability properties of second order delay integro-differential equations. Comput. Math. Appl. 56(12), 309-311 (2008)

8. Yenicerioglu, AF: The behavior of solutions of second order delay differential equations. J. Math. Anal. Appl. 332(2), 1278-1290 (2007)

9. Ashyralyev, A, Akca, H, Guray, U: Second order of accuracy difference scheme for approximate solutions of delay differential equations. Funct. Differ. Equ. 6(3-4), 223-231 (1999)

10. Ashyralyev, A, Akca, H: Stability estimates of difference schemes for neutral delay differential equations. Nonlinear Anal., Theory Methods Appl. 44(4), 443-452 (2001)

11. Ashyralyev, A, Akca, H, Yenicerioglu, AF: Stability properties of difference schemes for neutral differential equations. Differ. Equ. Appl. 3, 57-66 (2003)

12. Liu, J, Dong, P, Shang, G: Sufficient conditions for inverse anticipating synchronization of unidirectional coupled chaotic systems with multiple time delays. In: Chinese Control and Decision Conference (CCDC 2010), pp. 751-756 (2010)

13. Akca, H, Shakhmurov, VB, Arslan, G: Differential-operator equations with bounded delay. Nonlinear Times Dig. 2 179-190 (1989)

14. Sahmurova, A, Shakhmurov, VB: Parabolic problems with parameter occurring in environmental engineering. In: Ashyralyev, A, Lukashov, A (eds.) First International Conference on Analysis and Applied Mathematics (ICAAM 2012). AIP Conference Proceedings, vol. 1470, pp. 39-41 (2012)

15. Ashyralyev, A, Agirseven, D: Finite difference method for delay parabolic equations. In: International Conference on Numerical Analysis and Applied Mathematics (ICNAAM 2011). AIP Conference Proceedings, vol. 1389, pp. 573-576 (2011)

16. Agirseven, D: Approximate solutions of delay parabolic equations with the Dirichlet condition. Abstr. Appl. Anal. 2012, Article ID 682752 (2012)

17. Ashyralyev, A, Sobolevskii, PE: On the stability of the delay differential and difference equations. Abstr. Appl. Anal. 6(5) 267-297 (2001)

18. Ashyralyev, A, Sobolevskii, PE: New Difference Schemes for Partial Differential Equations. Birkhäuser, Basel (2004)

19. Di Blasio, G: Delay differential equations with unbounded operators acting on delay terms. Nonlinear Anal., Theory Methods Appl. 52(1), 1-18 (2003)

20. Ashyralyev, A, Sobolevskii, PE: The theory of interpolation of linear operators and the stability of difference schemes. Dokl. Akad. Nauk SSSR 275(6), 1289-1291 (1984) (in Russian)

21. Bazarov, MA: On the structure of fractional spaces. In: Proceedings of the XXVII All-Union Scientific Student Conference 'The Student and Scientific-Technological Progress', pp. 3-7. Gos. Univ., Novosibirsk (1989) (in Russian)

22. Triebel, H: Interpolation Theory, Function Spaces, Differential Operators. North-Holland, Amsterdam (1978)

23. Ashyralyev, A, Sobolevskii, PE: Well-Posedness of Parabolic Difference Equations. Operator Theory Advances and Applications. Birkhäuser, Basel (1994) 
24. Ashyralyev, A: Fractional spaces generated by the positive differential and difference operator in a Banach space. In: Tas, K, Tenreiro Machado, JA, Baleanu, D (eds.) Proceedings of the Conference 'Mathematical Methods and Engineering', pp. 13-22. Springer, Berlin (2007)

25. Ashyralyev, A, Akturk, S, Sozen, Y: Positivity of two-dimensional elliptic differential operators in Holder spaces. In: First International Conference on Analysis and Applied Mathematics (ICAAM 2012). AIP Conference Proceedings, vol. 1470, pp. 77-79 (2012)

26. Ashyralyev, A, Yaz, N: On structure of fractional spaces generated by positive operators with the nonlocal boundary value conditions. In: Agarwal, RF, Perera, K (eds.) Proceedings of the Conference Differential and Difference Equations and Applications, pp. 91-101. Hindawi Publishing Corporation, New York (2006)

27. Ashyralyev, A, Tetikoğlu, FS: The structure of fractional spaces generated by the positive operator with periodic conditions. In: First International Conference on Analysis and Applied Mathematics (ICAAM 2012). AIP Conference Proceedings, vol. 1470, pp. 57-60 (2012)

28. Ashyralyev, A, Agirseven, D: Approximate solutions of delay parabolic equations with the Neumann condition. In: International Conference on Numerical Analysis and Applied Mathematics (ICNAAM 2012). AIP Conference Proceedings, vol. 1479, pp. 555-558 (2012)

29. Ashyralyev, A, Agirseven, D: On convergence of difference schemes for delay parabolic equations. Comput. Math. Appl. 66(7), 1232-1244 (2013)

30. Ashyralyev, A, Agirseven, D: Well-posedness of delay parabolic difference equations. Adv. Differ. Equ. 2014, Article ID 18 (2014). doi:10.1186/1687-1847-2014-18

31. Solomyak, MZ: Estimation of norm of the resolvent of elliptic operator in spaces $L_{p}$. Usp. Mat. Nauk 15(6), 141-148 (1960) (in Russian)

32. Stewart, HB: Generation of analytic semigroups by strongly elliptic operators. Trans. Am. Math. Soc. 190, 141-162 (1974)

10.1186/1687-2770-2014-126

Cite this article as: Ashyralyev and Agirseven: Well-posedness of delay parabolic equations with unbounded operators acting on delay terms. Boundary Value Problems 2014, 2014:126

\section{Submit your manuscript to a SpringerOpen ${ }^{\circ}$ journal and benefit from:}

- Convenient online submission

- Rigorous peer review

- Immediate publication on acceptance

Open access: articles freely available online

- High visibility within the field

- Retaining the copyright to your article 